\title{
Quenching of Er(III) luminescence by ligand C-H vibrations: Implications for the use of erbium complexes in telecommunications
}

\author{
L. Winkless, R. H. C. Tan, and Y. Zheng \\ Department of Physics, Queen Mary, University of London, London E1 4NS, United Kingdom \\ M. Motevalli and P. B. Wyatt \\ School of Biological and Chemical Sciences, Queen Mary, University of London, London E1 4NS, \\ United Kingdom \\ W. P. Gillin ${ }^{\text {a) }}$ \\ Department of Physics, Queen Mary, University of London, London E1 4NS, United Kingdom
}

(Received 18 May 2006; accepted 16 July 2006; published online 14 September 2006)

\begin{abstract}
The authors have quantified the quenching of the luminescence lifetime of $\mathrm{Er}^{3+}$ ions in organic complexes due to the presence of $\mathrm{CH}$ vibrational oscillators as a function of their distance from the ion. They have shown that any hydrogen atoms within a sphere of at least $20 \AA$ from an erbium ion will cause sufficient quenching to prohibit its use in telecommunications applications. () 2006 American Institute of Physics. [DOI: 10.1063/1.2345909]
\end{abstract}

Erbium-doped glasses are widely used for telecommunications due to the sharp intra-atomic ${ }^{4} I_{13 / 2} \rightarrow{ }^{4} I_{15 / 2}$ transition in the $4 f$ orbital which result in an emission at $\sim 1.5 \mu \mathrm{m}$, which is the low loss window for silica optical fiber. If erbium is incorporated into organic hosts then the organic ligands can act as photosensitizers, resulting in much higher absorption cross sections, with broader absorption bands than for the free $\mathrm{Er}^{3+}$ ion. ${ }^{1-4}$ However, ligands and coordinated solvent molecules usually contain $\mathrm{C}-\mathrm{H}$ and $\mathrm{O}-\mathrm{H}$ bonds that can cause vibrational quenching of electronically excited erbium; this limits any application of such complexes in infrared emitting devices. Deuteration or fluorination of hydrogen-containing ligands, together with exclusion of coordinated water, can extend the lifetime of infrared luminescence from lanthanide complexes. ${ }^{5} \mathrm{Nd}(\mathrm{III})$ has been a subject of particular study; however, there have been little data in actually quantifying the extent of the quenching. A recent paper by Quochi et al. ${ }^{6}$ has proposed a model for calculating the degree of quenching based on the assumption of a continuous distribution of $\mathrm{CH}$ oscillators which makes good predictions of the degree of quenching for systems where there are large amounts of $\mathrm{CH}$ or $\mathrm{OH}$ bonds present. As the authors state, the recent work by Mancino et al. ${ }^{7}$ where it has been shown that an evaporated thin film of $\operatorname{Er}(\mathrm{F} \text {-tpip })_{3}$, where F-tpip is the perfluorinated imidodiphosphinate $\left[\left(\mathrm{C}_{6} \mathrm{~F}_{5}\right)_{2} \mathrm{P}(\mathrm{O})\right]_{2} \mathrm{~N}^{-}$, having a luminescence lifetime of $224 \mu \mathrm{s}$ does not fit with their model and they suggest that the discrepancy is probably due to residual hydrogen-containing impurities.

In order to quantify the effect of $\mathrm{O}-\mathrm{H}$ and $\mathrm{C}-\mathrm{H}$ bonds on the radiative lifetime of $\mathrm{Er}^{3+}$ ions and to determine the spatial distance over which deexcitation can occur we have used the tris complex $\operatorname{Er}(\mathrm{HFA})_{3}$ and the tetrakis complex $[\mathrm{Cs}]\left[\mathrm{Er}(\mathrm{HFA})_{4}\right] \quad(\mathrm{H}-\mathrm{HFA}=1,1,1,5,5,5$-hexafluoro- $2,4-$ pentanedione) to study the effect of removing water from the coordination sphere of the erbium ion. We have also investigated the effect of replacing the single hydrogen atom on the 3 position of the ligand with deuterium. We have shown that

${ }^{a)}$ FAX: +44020 8981 9465; electronic mail: w.gillin@qmul.ac.uk by removing $\mathrm{OH}$ and $\mathrm{CH}$ bonds to increasing distances from an $\mathrm{Er}^{3+}$ ion we can obtain a three order of magnitude increase in the radiative lifetime and that the presence of a $\mathrm{CH}$ bond within a radius of $\sim 20 \AA$ of an $\mathrm{Er}^{3+}$ ion can still be an effective deexcitation site for the $\mathrm{Er}^{3+}$ ion. These studies provide vital data for the future design of efficient lanthanide emitters using organic ligands.

[Er(HFA $)_{3}\left(\mathrm{H}_{2} \mathrm{O}\right)_{2}$ ] was prepared according to Ref. 8 and recrystallized from toluene to give large pink needles, $\mathrm{mp}$ $(118-121){ }^{\circ} \mathrm{C}$ which were characterized by $\mathrm{CH}$ analysis, high resolution mass spectrometry, IR spectroscopy and single crystal x-ray diffraction (XRD). $[\mathrm{Cs}]\left[\operatorname{Er}(\mathrm{HFA})_{4}\right]$ was prepared by mixing aqueous CsHFA with the calculated amount of aqueous erbium(III) chloride; the pink precipitate was recrystallized from aqueous methanol to give pink needles which sublimed on heating above $200{ }^{\circ} \mathrm{C}$ and were characterized by $\mathrm{CH}$ analysis, high resolution mass spectrometry, IR spectroscopy, and single crystal x-ray diffraction and were found to be isomorphous with $[\mathrm{Cs}]\left[\mathrm{Eu}(\mathrm{HFA})_{4}\right]^{9}{ }^{9}$ H-HFA was deuterated according to Ogoshi and Nakamoto, ${ }^{10}$ then used to prepare deuterated $[\mathrm{Cs}]\left[\operatorname{Er}(\mathrm{HFA})_{4}\right]$ in $\mathrm{D}_{2} \mathrm{O}$ solution; the precipitated product was then recrystallized from $\mathrm{CH}_{3} \mathrm{OD}-\mathrm{D}_{2} \mathrm{O}$. Laser desorption/ionization mass spectroscopy (Bruker Daltonics Autoflex) on the resultant material showed that we had achieved $98 \% \pm 1 \%$ deuteration.

Photoluminescence from the complexes was excited using $\sim 7 \mathrm{~ns}$ pulses, at an excitation wavelength of $520 \mathrm{~nm}$, from a Continuum Panther optical parametric oscillator pumped with a Surelite I laser. This wavelength was chosen to provide direct excitation into the ${ }^{4} S_{3 / 2}$ level of the erbium ion which rapidly decays to the ${ }^{4} I_{13 / 2}$ level with a measured decay time of the order of $25 \mathrm{~ns}$. The luminescence was dispersed in a Triax 550 spectrometer and detected using a Hamamatsu R5509-72 infrared photomultiplier tube.

Lifetime data for the ${ }^{4} I_{13 / 2} \rightarrow{ }^{4} I_{15 / 2}$ transition were recorded at the peak of the photoluminescence spectra $(1542 \mathrm{~nm})$ at a temperature of $300 \mathrm{~K}$ and fitted with either a one or two exponential decay model using a MarquardtLevenberg algorithm to find the best fit. Table I shows the results of the lifetime fits to five samples. Three samples 
TABLE I. Measured lifetimes of the $1542 \mathrm{~nm}$ emission from the $\mathrm{Er}^{3+}$ ${ }^{4} I_{13 / 2} \rightarrow{ }^{4} I_{15 / 2}$ transition. The figures in parentheses are the percentage contribution from each component.

\begin{tabular}{lcc}
\hline \hline & \multicolumn{1}{c}{$\tau_{1}$} & $\tau_{2}$ \\
\hline$\left[\operatorname{Er}(\mathrm{HFA})_{3}\left(\mathrm{H}_{2} \mathrm{O}\right)_{2}\right]$ dried & $132 \mathrm{~ns}(17 \%)$ & \multirow{2}{*}{$1.8 \mu \mathrm{s}(83 \%)$} \\
{$\left[\operatorname{Er}(\mathrm{HFA})_{3}\left(\mathrm{H}_{2} \mathrm{O}\right)_{2}\right]$ hydrated } & $100 \mathrm{~ns}(100 \%)$ & \\
{$[\mathrm{Cs}]\left[\mathrm{Er}(\mathrm{HFA})_{4}\right]$} & $1.8 \mu \mathrm{s}(100 \%)$ & \\
{$[\mathrm{Cs}]\left[\mathrm{Er}(\mathrm{HFA})_{4}\right]$ in $\mathrm{CD}_{3} \mathrm{OD}$} & $1.6 \mu \mathrm{s}(100 \%)$ & \\
{$[\mathrm{Cs}]\left[\operatorname{Er}(\mathrm{HFA})_{4}\right] 98 \%$ deuterated } & $11 \mu \mathrm{s}(96.4 \%)$ & $106 \mu \mathrm{s}(3.6 \%)$ \\
\hline \hline
\end{tabular}

show single exponential decay, the $\left[\operatorname{Er}(\mathrm{HFA})_{3}\left(\mathrm{H}_{2} \mathrm{O}\right)_{2}\right]$, the $[\mathrm{Cs}]\left[\mathrm{Er}(\mathrm{HFA})_{4}\right]$, and the $[\mathrm{Cs}]\left[\mathrm{Er}(\mathrm{HFA})_{4}\right]$ dissolved in methanol- $\mathrm{d}_{4}$. The presence of a single exponential decay suggests that all the $\mathrm{Er}^{3+}$ ions are in identical local environments.

For the $\left[\operatorname{Er}(\mathrm{HFA})_{3}\left(\mathrm{H}_{2} \mathrm{O}\right)_{2}\right]$ sample that had been dried in vacuo at 1 mbar over $\mathrm{P}_{2} \mathrm{O}_{5}$ there are two lifetime components, a major one with a lifetime of $1.8 \mu \mathrm{s}(83 \%)$ and a minor one with a lifetime of $132 \mathrm{~ns}(17 \%)$. The $132 \mathrm{~ns}$ component is due to the dramatic deexcitation effect of the water molecules in the erbium coordination sphere, where each hydrogen is only $\sim 2.9 \AA$ (determined from the XRD data) from the $\mathrm{Er}^{3+}$ ion. The $1.8 \mu$ s component is probably due to the presence of some anhydrous tris salt being formed during recrystallization and drying. Samples of this material were left next to an open beaker of water for $24 \mathrm{~h}$ in a sealed container. This was sufficient to fully hydrate the material so that only a pure $100 \mathrm{~ns}$ lifetime was observed. The slightly higher lifetime for the fast component in the anhydrous sample is probably due to some of the ions having only one water molecule in their coordination sphere as opposed to the two found in the hydrated sample. This hydrated sample was sublimed under a $10^{-7}$ mbar vacuum in a Kurt J. Lesker SPECTROS deposition system and the resulting material was found to be very similar to the hydrated sample with $\sim 85 \%$ with a lifetime of $100 \mathrm{~ns}$ and $\sim 15 \%$ with a lifetime of $0.5 \mu \mathrm{s}$. The $0.5 \mu \mathrm{s}$ lifetime is probably shorter than that seen in the original "anhydrous" salt due to the low number of ions in this environment resulting in even the erbium ions in an anhydrous environment having water molecules in next nearest neighbor positions. This demonstrates that the coordinated water is difficult to entirely remove by sublimation which is in agreement with the findings of Richardson and Sievers. ${ }^{11}$

The $[\mathrm{Cs}]\left[\mathrm{Er}(\mathrm{HFA})_{4}\right]$ sample was measured both before and after sublimation and no difference in the measured lifetime was observed. The lifetime of $1.8 \mu \mathrm{s}$, although more than an order of magnitude greater than for the $\left[\operatorname{Er}(\mathrm{HFA})_{3}\left(\mathrm{H}_{2} \mathrm{O}\right)_{2}\right.$ ], demonstrates that even four $\mathrm{CH}$ bonds at a distance of $\sim 4.7 \AA$ (determined from the XRD data) from the $\mathrm{Er}^{3+}$ ion will have a dramatic effect of the deexcitation of the ion. For the $[\mathrm{Cs}]\left[\mathrm{Er}(\mathrm{HFA})_{4}\right]$ the sample was measured within $5 \mathrm{~min}$ of dissolving in order to reduce the probability of significant exchange of the hydrogen on the ligands. The very small decrease in the lifetime from the solid $[\mathrm{Cs}]\left[\operatorname{Er}(\mathrm{HFA})_{4}\right]$ sample to that dissolved in methanol- $\mathrm{d}_{4}$ suggests that the lifetime of the $\mathrm{Er}^{3+}$ ions in the $\left[\mathrm{Er}(\mathrm{HFA})_{4}\right]^{-}$ anion is primarily affected by the $\mathrm{CH}$ bonds in each of the coordinated HFA ligands with only a small contribution from $\mathrm{CH}$ bond in the surrounding HFA ligands. From the $\mathrm{x}$-ray crystallography these hydrogen atoms are at $\sim 4.7 \AA$ from

Downloaded 15 Sep 2006 to 138.37.52.15. Redistribution subject

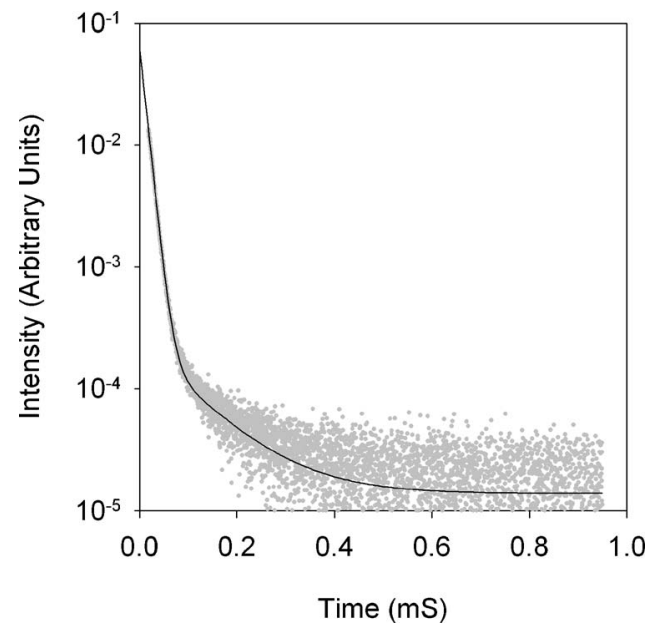

FIG. 1. Lifetime data for the $98 \%$ deuterated $[\mathrm{Cs}]\left[\operatorname{Er}(\mathrm{HFA})_{4}\right]$ sample recorded at a wavelength of $1542 \mathrm{~nm}$.

the $\mathrm{Er}^{3+}$ ion. To investigate the role of these hydrogen atoms more fully we examined our $98 \%$ deuterated $[\mathrm{Cs}]\left[\mathrm{Er}(\mathrm{HFA})_{4}\right]$. The luminescence for this material showed two lifetimes with the major component $(96.4 \%)$ having a lifetime of $11 \mu \mathrm{s}$ and with $3.6 \%$ having a lifetime of $\sim 106 \mu$ s (Fig. 1). It should be stressed that the low intensity of this component results in a significant error on the value of this lifetime. The fact that one component has a lifetime of $\sim 106 \mu$ s implies that some of the $\mathrm{Er}^{3+}$ ions are in an environment with only a relatively weak deexcitation process occurring. If we assume that this is due to $\mathrm{Er}^{3+}$ ions that are in positions with no hydrogen in their nearest neighbor positions we can calculate that with $98 \%$ deuteration $3.6 \%$ of the $\mathrm{Er}^{3+}$ ions will be in sites where the $\sim 160$ nearest hydrogen sites are all fully deuterated. From the crystallographic data it can be seen that this corresponds to a sphere of radius $\sim 20 \AA$ around the $\mathrm{Er}^{3+}$ ion and is approximately equivalent to each of the 40 next nearest $\left[\operatorname{Er}(\mathrm{HFA})_{4}\right]^{-}$anions being fully deuterated. Given the $98 \%$ deuteration level in this sample there is a $92 \%$ probability of an erbium being in a fully deuterated anion and a 7\% probability of an anion having three deuteriums and one hydrogen. As we could only resolve one other lifetime component at $11 \mu \mathrm{s}$ it suggests that in both of these environments the lifetime is dominated by the presence of hydrogen atoms in the next nearest neighbor sites.

As the natural radiative lifetime $\tau_{R}$ of the erbium will be of the order of milliseconds we can take the measured lifetime $\tau_{M}$ to be a good approximation of the sum of the rate

$$
\frac{1}{\tau_{M}}=\frac{1}{\tau_{R}}+\sum k
$$

constants, $k$, for all the quenching mechanisms present.

As there is a Förster energy transfer ${ }^{12}$ between the vibrational oscillator and the erbium the transfer will be inversely proportional to the sixth power of the distance between them. For molecules with a short lifetime we can therefore, to a first order approximation, consider the quenching to be dominated by the nearest neighbor oscillators. From the $[\mathrm{Cs}]\left[\operatorname{Er}(\mathrm{HFA})_{4}\right]$ lifetime this allows us to place an upper limit on the rate constant per $\mathrm{CH}$ bond on the anion (i.e., at a distance of $4.7 \AA$ from the $\mathrm{Er}^{3+}$ ion) of $\sim 140000 \mathrm{~s}^{-1}$. This would give a lifetime for an $\mathrm{Er}^{3+}$ ion with one hydrogen and AIP license or copyright, see http://apl.aip.org/apl/copyright.jsp 
three deuterium atoms in the nearest neighbor positions of $\sim 7 \mu \mathrm{s}$, which is of the correct order; the actual value of $11 \mu$ s shows that the presence of $\mathrm{CH}$ bonds in the second nearest neighbor shell does play some role in the quenching and that the actual rate constant for a $\mathrm{CH}$ bond in the anion is probably less than $91000 \mathrm{~s}^{-1}$. This should be compared to the maximum value for a $\mathrm{CD}$ bond in the same position which is $\sim 2400 \mathrm{~s}^{-1}$ determined from the $106 \mu$ s lifetime. It should be stressed that it is not clear whether the $106 \mu \mathrm{s}$ lifetime is dominated by the quenching to the $\mathrm{CD}$ oscillators in the nearest neighbor positions or due to more distant $\mathrm{CH}$ oscillators.

These results demonstrate that the nearest neighbor $\mathrm{CH}$ oscillators are by far the biggest contributor to the quenching of erbium ions in organic hosts which corresponds to the $R_{\min }$ value in the theory proposed by Quochi et al., ${ }^{6}$ the role of the more distant oscillators is only of secondary importance. These results also show that to produce erbiumcontaining molecules where the lifetime of the erbium is not being dominated by quenching to hydrogen-related vibrational modes in the surrounding ligands requires the removal of all hydrogen from a distance of at least $20 \AA$ from the $\mathrm{Er}^{3+}$ ions, for a $100 \mu \mathrm{s}$ lifetime. This is still far below the expected radiative recombination lifetime for the ion despite the average $\mathrm{CH}$ concentration in this sample $\left(\sim 10^{3} \AA^{-1}\right)$ which is too low for us to observe by IR absorption. The results also demonstrate that although deuterating ligands can dramatically improve the $\mathrm{Er}^{3+}$ luminescence lifetime, the quenching due to any $\mathrm{CH}$ bonds which are still present is so strong that deuteration is not a viable solution and this means that fully halogenated ligands will have to be used. It also shows that it will be necessary to produce molecules where there is no hydrogen-containing solvent within the crystal structure which suggests that solution processing as a means of producing devices may be problematic without the use of purely halogenated solvents. Furthermore due to the extreme sensitivity to hydrogen it is also probable that even in fully halogenated systems repeated vacuum sublimation as a purification step may well be necessary to obtain optimized lifetimes.

The authors thank the EPSRC National Crystallography Service, Southampton for data collection and the EPSRC National Mass Spectrometry Service, Swansea for mass spectra.

${ }^{1}$ L. H. Slooff, A. Polman, M. P. Oude Wolbers, F. C. J. M. van Veggel, D. N. Reinhoudt, and J. W. Hofstraat, J. Appl. Phys. 83, 497 (1998).

${ }^{2}$ R. J. Curry and W. P. Gillin, Appl. Phys. Lett. 75, 1380 (1999).

${ }^{3}$ R. J. Curry and W. P. Gillin, Curr. Opin. Solid State Mater. Sci. 5, 481 (2002).

${ }^{4}$ J.-C. G. Bünzli and C. Piguet, Chem. Soc. Rev. 34, 1048 (2005).

${ }^{5}$ Y. Hasegawa, Y. Wada, and S. Yanagida, J. Photochem. Photobiol. C 5, 183 (2004).

${ }^{6}$ F. Quochi, R. Orru, F. Cordella, A. Mura, G. Bongiovanni, F. Artizzu, P. Deplano, M. L. Mercuri, L. Pilia, and A. Serpe, J. Appl. Phys. 99, 053520 (2006).

${ }^{7}$ G. Mancino, A. J. Ferguson, A. Beeby, N. J. Long, and T. S. Jones, J. Am. Chem. Soc. 127, 524 (2005).

${ }^{8}$ M. F. Richardson, W. F. Wagner, and D. E. Sands, J. Inorg. Nucl. Chem. 30, 1275 (1968).

${ }^{9}$ J. H. Burns and M. D. Danford, Inorg. Chem. 8, 1780 (1969).

${ }^{10}$ H. Ogoshi and K. Nakamoto, J. Chem. Phys. 45, 3113 (1966).

${ }^{11}$ M. F. Richardson and R. E. Sievers, Inorg. Chem. 10, 498 (1971).

${ }^{12}$ T. Förster, Ann. Phys. 2, 55 (1948). 